\title{
Fast time domain modeling of surface scattering from reflectors and diffusers
}

\author{
Trevor J. Cox \\ Acoustics Research Centre, University of Salford, Salford, United Kingdom \\ t.j.cox@salford.ac.uk
}

\begin{abstract}
Time-domain prediction models have been developed for auditorium reflectors and room acoustic diffusers. The models are timedomain equivalents of the single-frequency formulations that exploit the Kirchhoff boundary conditions. Consequently, they are approximate, wave-based solutions to the Kirchhoff integral equation using surface meshes. The new time-domain formulations are validated by comparison to their frequency-domain equivalents for three different surfaces: a plane surface, a curved reflector, and a Schroeder diffuser. In terms of computation time and accuracy, the new models lie between the finite difference time domain and geometric room models.

(C) 2015 Acoustical Society of America

[NX]

Date Received: February 6, 2015 Date Accepted: May 12, 2015
\end{abstract}

\section{Introduction}

A variety of models exist to predict the reflection and diffraction from architectural structures such as noise barriers, stage canopies and room acoustic diffusers. ${ }^{1,2}$ In the timedomain, finite difference time domain (FDTD) is arguably the most popular wave-based approach. As a method that uses a volumetric mesh, however, calculation times can become excessively long. Green's theorem enables the linear wave equation to be written as a boundary integral equation, removing the need to use a volumetric mesh. This leads to techniques such as boundary element methods (BEMs) based on the HelmholtzKirchhoff integral equation. A BEM requires the solution of a potentially large number of simultaneous equations and so can also be slow to compute. For this reason, there are a number of single-frequency approximate solutions analogous to Kirchhoff, Fresnel, and Fraunhofer models used in optics. These frequency-domain models are, however, inefficient for calculating impulse responses. Consequently, this paper derives timedomain formulations that are equivalent to single-frequency methods that exploit the Kirchhoff boundary conditions.

\section{Theory}

First, the formulation for non-absorbing, thin reflectors such as curved or flat surfaces is derived using the geometry shown in Fig. 1. The sound field at receiver point $r$ and time $t$ in the vicinity of a scatterer $S$ is represented by the pressure $p_{t}(\boldsymbol{r}, t)=p_{i}+p_{s}$, where $p_{i}$ represents the sound traveling directly from the source to the receiver along vector $\boldsymbol{r}_{\boldsymbol{d}}$, and $p_{s}$ the sound scattered off the surface. The pressure can be found from the Kirchhoff integral equation ${ }^{3}$

$$
p_{t}(\boldsymbol{r}, t)=p_{i}(\boldsymbol{r}, t)+\iint_{S}\left[p_{t}\left(\boldsymbol{r}_{\boldsymbol{s}}, t\right) * \hat{\boldsymbol{n}} \cdot \nabla g\left(\boldsymbol{r} \mid \boldsymbol{r}_{\boldsymbol{s}}, t\right)-g\left(\boldsymbol{r} \mid \boldsymbol{r}_{\boldsymbol{s}}, t\right) * \hat{\boldsymbol{n}} \cdot \nabla p_{t}\left(\boldsymbol{r}_{\boldsymbol{s}}, t\right)\right] d s,
$$

where $\boldsymbol{r}_{\boldsymbol{s}}$ is a point on that surface; $\boldsymbol{n}$ is the normal to surface, and $*$ denotes convolution. $g()$ is the time-domain Green's function that describes how the pressure created by an instantaneous point source at time $t=0$, travels to another location. It is given by 


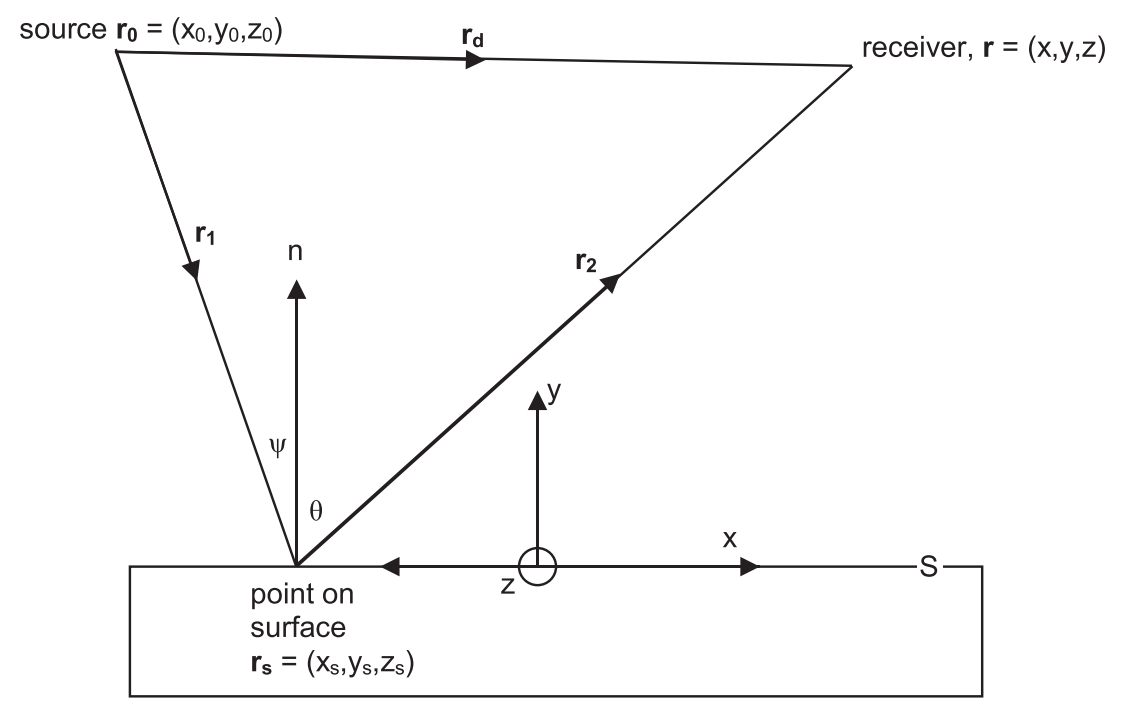

Fig. 1. The geometry used in the prediction models.

$$
g\left(\boldsymbol{r}_{\boldsymbol{a}} \mid \boldsymbol{r}_{\boldsymbol{b}}, t\right)=\frac{\delta\left(t-R_{a b} / c\right)}{4 \pi R_{a b}},
$$

where $\boldsymbol{R}_{\boldsymbol{a}}=\boldsymbol{r}_{\boldsymbol{a}}-\boldsymbol{r}_{\boldsymbol{b}}$ and $\delta()$ is a Dirac delta function. As the surface material is considered to be hard and non-absorbing, the last term in the integral in Eq. (1), which represents the normal component of the particle velocity, is for now assumed to be zero. The incident field is taken to be created by a monopole with time-dependent amplitude, $F(t)$,

$$
p_{i}(\boldsymbol{r}, t)=F(t) * g\left(\boldsymbol{r} \mid \boldsymbol{r}_{\mathbf{0}}, t\right)=F\left(t-r_{d} / c\right),
$$

where $\boldsymbol{r}_{\boldsymbol{d}}$ is the vector from the point source to the receiver point and $c$ the speed of sound.

For non-absorbing materials, the Kirchhoff boundary conditions simply states that there will be a doubling of the incident pressure at the surface, ${ }^{1}$ i.e., $p_{t}=2 p_{i}$ and $\hat{\boldsymbol{n}} . \nabla p_{t}\left(\boldsymbol{r}_{\boldsymbol{s}}, t\right)=0$. Learning from the frequency domain models that use this boundary condition, it would be anticipated that inaccuracies will arise when the surface: (i) has significant corrugation that can then create second order reflections and (ii) is small compared to wavelength, because edge diffraction is not fully modeled.

To solve the integration, the surface is discretized into $N$ elements that are small compared to wavelength, so the pressure can be approximated to be constant across the elements. Applying this to the integral equation with the source function and boundary condition gives

$$
p_{t}(\boldsymbol{r}, t)=F(t) * g\left(\boldsymbol{r} \mid \boldsymbol{r}_{\mathbf{0}}, t\right)+2 \sum_{n=1}^{N} \cos (\theta) \iint F(t) * g\left(\boldsymbol{r}_{\boldsymbol{s}} \mid \boldsymbol{r}_{\mathbf{0}}, t\right) * \frac{\partial g\left(\boldsymbol{r} \mid \boldsymbol{r}_{\boldsymbol{s}}, t\right)}{\partial r_{2}} d s,
$$

where $\theta$ is the angle of reflection relative to the surface differential normal and $\boldsymbol{r}_{2}$ the vector from the point on the surface to the receiver. The last term includes the differential of the Green's function, which is the differential of a delta function. This is dealt by using the following identity that exploits the quotient rule and includes a far field approximation so that the $1 / r_{2}$ term can be moved outside the differential: ${ }^{4}$ 


$$
F(t) * \frac{\partial g\left(\boldsymbol{r} \mid \boldsymbol{r}_{s}, t\right)}{\partial r_{2}} \approx-\frac{1}{4 \pi r_{2} c} \dot{F}\left(t-\frac{r_{2}}{c}\right),
$$

where a dot over the function signifies the time derivative. Substituting Eq. (5) into Eq. (4) yields

$$
p_{t}(\boldsymbol{r}, t)=F(t) * g\left(\boldsymbol{r} \mid \boldsymbol{r}_{\mathbf{0}}, t\right)-2 \sum_{n=1}^{N} \frac{\cos (\theta)}{4 \pi r_{2} c} \iint g\left(\boldsymbol{r}_{\boldsymbol{s}} \mid \boldsymbol{r}_{\mathbf{0}}, t\right) * \dot{F}\left(t-\frac{r_{2}}{c}\right) d s .
$$

As it has already been assumed that this is a far field model, the term in $1 / r_{2}$ can be moved outside the integration. A Gaussian function is chosen as the excitation function, as it is commonly used in many time-domain models ${ }^{5}$

$$
F(t)=\frac{1}{\sqrt{2 \pi} \sigma} e^{-\left(t^{2} / 2 \sigma^{2}\right)}
$$

The following differential is also required:

$$
\dot{F}\left(t-\frac{r_{2}}{c}\right)=\frac{\left(t-\frac{r_{2}}{c}\right)}{c \sigma^{2}} F\left(t-\frac{r_{2}}{c}\right) .
$$

Substituting Eqs. (7) and (8) into Eq. (6) yields

$$
\begin{aligned}
p_{t}(\boldsymbol{r}, t)= & \frac{1}{4 \sqrt{2} \pi^{3 / 2} \sigma r_{d}} e^{-\left(t-r_{d} / c\right)^{2} / 2 \sigma^{2}} \\
& -\sum_{n=1}^{N} \frac{\cos (\theta)}{8 \sqrt{2} \pi^{5 / 2} r_{1} r_{2} \sigma^{3} c} \iint \delta\left(t-r_{1} / c\right) *\left(t-\frac{r_{2}}{c}\right) e^{-\left(t-r_{2} / c\right)^{2} / 2 \sigma^{2}} d s,
\end{aligned}
$$

where $1 / r_{1}$ has been moved outside the integration as this is a far field solution.

The integration in Eq. (9) is treated as a simple summation and the convolution with the delta function is resolved. If each element has area $\Delta s$, then the pressure is

$$
\begin{aligned}
p_{t}(\boldsymbol{r}, t)= & \frac{1}{4 \sqrt{2} \pi^{3 / 2} \sigma r_{d}} e^{-\left(t-r_{d} / c\right)^{2} / 2 \sigma^{2}} \\
& -\sum_{n=1}^{N} \frac{\cos (\theta) \Delta s}{8 \sqrt{2} \pi^{5 / 2} r_{1} r_{2} \sigma^{3} c}\left(t-\frac{r_{1}+r_{2}}{c}\right) e^{-\left(t-\left(r_{1}+r_{2}\right) / c\right)^{2} / 2 \sigma^{2}} .
\end{aligned}
$$

The plane and curved reflectors are assumed to be thin. The front face is discretized into elements that are smaller than a sixth of a wavelength for the highest frequency of interest. An important detail of note is that the accurate modeling of arrival times of the Gaussian pulses is vital and so oversampling is used. ${ }^{6}$ Equation (10) is calculated with a sampling frequency at least ten times the highest frequency of interest and the samples of each Gaussian pulse are moved to the nearest sample point.

The first model of a Schroeder diffuser needs an addition to Eq. (10). A Schroeder diffuser, such as the quadratic residue diffuser (QRD) shown as an inset in Fig. 2(d), is made up of a set of wells that cause the sound reflecting from the surface to be delayed as plane waves propagate down and back up each well. ${ }^{7}$ A common approximate representation is to consider the diffuser as a thin flat surface that has a reflection coefficient that models the effects of the plane wave propagation within the wells. A similar approach can be taken in the time domain and has previously been used for TBEM ${ }^{3}$ and FDTD. ${ }^{8}$ In the proposed technique, the diffuser is modeled as a flat surface, but the reflections are delayed by the time it takes the sound waves to propagate within the wells assuming no losses. If the $n$th element is at the mouth of a well of depth $d_{n}$ then the scattered pressure in Eq. (10) is modified to 


$$
p_{s}(\boldsymbol{r}, t)=-\sum_{n=1}^{N} \frac{\cos (\theta) \Delta s}{8 \sqrt{2} \pi^{5 / 2} r_{1} r_{2} \sigma^{3} c}\left(t-\frac{2 d_{n}+r_{1}+r_{2}}{c}\right) e^{-\left(t-\left(2 d_{n}+r_{1}+r_{2}\right) / c\right)^{2} / 2 \sigma^{2}} .
$$

Equation (11) is equivalent to common models used in the frequency domain (e.g., Ref. 7) and will be described as the Fraunhofer model. This representation is expected to be suitable under similar conditions that the analogous frequency-domain model works: (i) the frequency content of the Gaussian pulse must be low enough that plane wave propagation in the wells dominates; (ii) the radiation coupling between the wells has to be small, and (iii) the radiation impedance of each well must be small.

A comparison of Eqs. (1) and (11) show, however, that the approximations in the derivation has removed some effects that arise for oblique incident sound, because there are no terms that explicitly have the angle of incidence, $\psi$. A more precise model needs to include the last term of Eq. (1). For this, the Kirchhoff Boundary condition also needs to be more completely considered. For a Schroeder diffuser, the pressure at the well entrance can be approximated as $p_{t}(\boldsymbol{r}, t)=p_{i}(\boldsymbol{r}, t)+p_{i}\left(\boldsymbol{r}, t-2 d_{n} / c\right)$. The first term represents an incident wave traveling with an angle of $-\psi$ to the normal. The second term is the wave reflected from the surface at an angle of $\psi^{\prime}$ to the normal. Following the normal rules of refraction, for many surfaces, $\psi^{\prime}=\psi$ would be an appropriate assumption. But for the narrow wells in a Schroeder diffuser, a better approximation might be that the reflected waves that reradiate from the wells travel parallel to the surface normal, $\psi^{\prime}=0$. Applying this to Eq. (1) and simplifying yields the following for the scattered pressure:
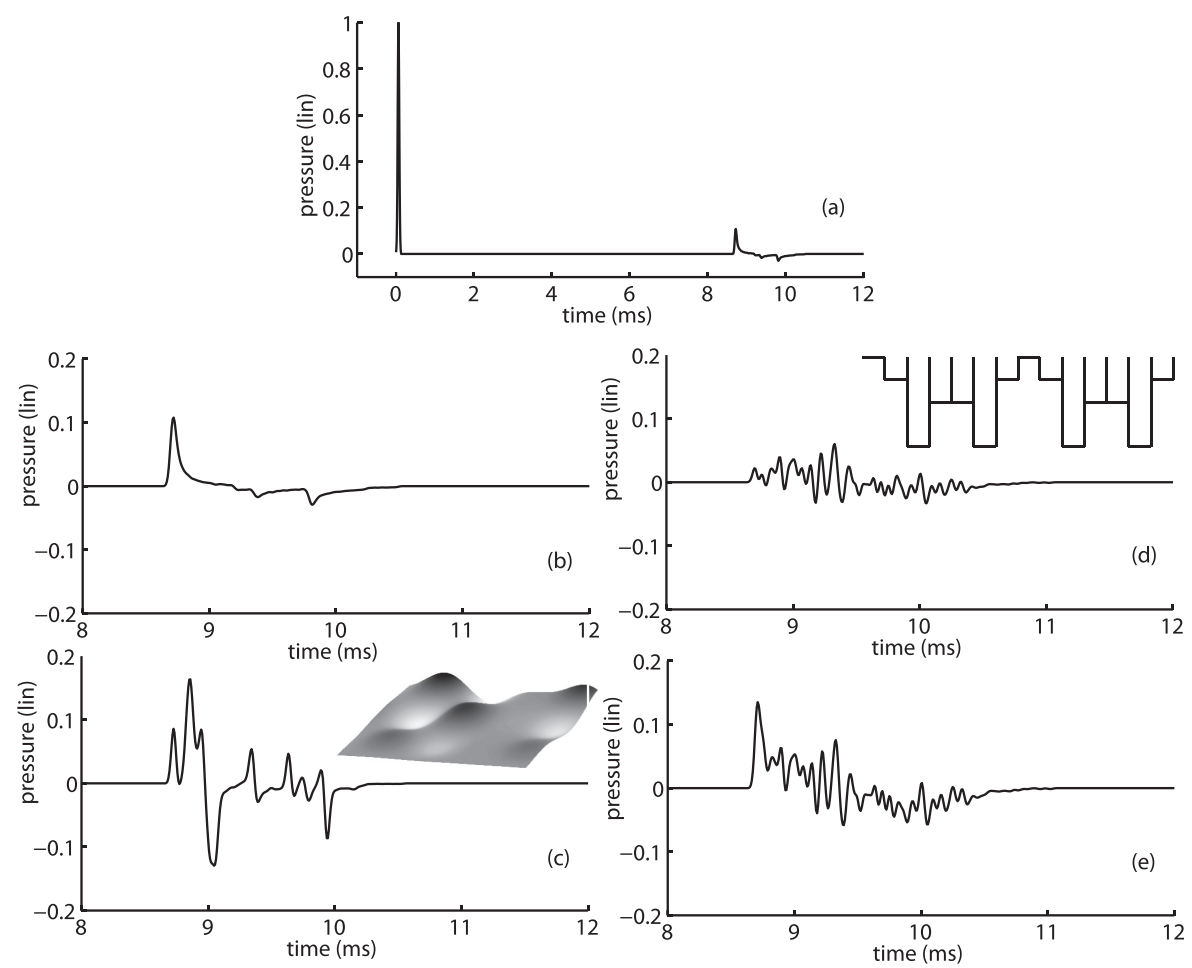

Fig. 2. Impulse response for (a) plane surface with both direct and reflected sound shown; (b) plane surface, reflection only; (c) curved surface, reflection only; (d) Schroeder diffuser, reflection only, Fraunhofer model; and (e) Schroeder diffuser, reflection only, Kirchhoff model. The peak of the direct sound has been set to unity for the plots. The insets in (c) and (d) illustrate two of the surface shapes. 


$$
\begin{aligned}
p_{s}(r, t)= & \frac{-\Delta s}{16 \sqrt{2} \pi^{5 / 2} r_{1} r_{2} c \sigma^{3}} \sum\left\{\left(t-\frac{r_{1}+r_{2}}{c}\right) e^{-\left(t-\left(r_{1}+r_{2}\right) / c\right)^{2} / 2 \sigma^{2}}(\cos (\theta)-\cos (\psi))\right. \\
& \left.+\left(t-\frac{r_{1}+r_{2}+2 d_{n}}{c}\right) e^{-\left(t-\left(r_{1}+r_{2}+2 d_{n}\right) / c\right)^{2} / 2 \sigma^{2}}\left(\cos (\theta)+\cos \left(\psi^{\prime}\right)\right)\right\} .
\end{aligned}
$$

This will be referred to as the Kirchhoff model, and is implemented in a similar manner to the previous models.

\section{Validation}

The results section include example predictions for various samples. The geometry chosen for the illustration were picked at random. The surfaces were all $1.4 \times 1 \mathrm{~m}$ in size. The curved surface was such that the difference between the minimum and maximum corrugation was $19 \mathrm{~cm}$. The shape of the corrugated surface was formed by adding some randomly chosen harmonics to create a wavy shape [see inset in Fig. 2(c)]. A one-dimensional quadratic residue diffuser was made based on the prime number 7 with the depth sequence along the $x$-direction. There were ten periods and the well width was $2 \mathrm{~cm}$. The design frequency was $1000 \mathrm{~Hz}$. The following $(x, y, z)$ coordinates for the oblique source and receiver positions were chosen: $(-2.5,4,-1)$ and $(4,3,1) \mathrm{m}$, respectively. Predictions were carried out up to $8 \mathrm{kHz}$. A sampling frequency of $128 \mathrm{kHz}$ was used and there were eight elements per minimum wavelength for the surface discretization.

To check that the new models were correct, the scattering was compared to standard single-frequency models. These are evaluations of the Helmholtz-Kirchhoff Integral Equation after the Kirchhoff boundary conditions had been applied. The frequency domain equivalent of Eqs. (10) and (11) is Eq. (8.26) from Ref. 1 with the surface reflection coefficient $R\left(\boldsymbol{r}_{s}\right)$ explicitly stated as follows:

$$
p_{t}(\boldsymbol{r})=G\left(\boldsymbol{r} \mid \boldsymbol{r}_{\mathbf{0}}\right)-i k \iint_{S} R\left(\boldsymbol{r}_{\boldsymbol{s}}\right) G\left(\boldsymbol{r}_{\boldsymbol{s}} \mid \boldsymbol{r}_{\mathbf{0}}\right) \cos (\theta) d s,
$$

where $k$ is the wavenumber and the frequency domain Green's function is $G\left(\boldsymbol{r}_{\boldsymbol{a}} \mid \mathbf{r}_{\mathbf{b}}\right)=\exp \left(-i k R_{a b}\right) / 4 \pi R_{a b}$. The frequency domain equivalent of Eq. (12) for the scattered pressure from a Schroeder diffuser is Eq. (8.25) from Ref. 1,

$$
p_{s}(\boldsymbol{r})=-i k \iint G\left(\boldsymbol{r}_{\boldsymbol{s}} \mid \boldsymbol{r}_{\mathbf{0}}\right) G\left(\mathbf{r} \mid \boldsymbol{r}_{\boldsymbol{s}}\right)\left[(\cos (\theta)-\cos (\psi))+\left(\cos (\theta)+\cos \left(\psi^{\prime}\right)\right) R\left(\boldsymbol{r}_{\boldsymbol{s}}\right)\right] d s .
$$

\section{Results}

Figure 2 shows the pressure vs time for the three surfaces tested using the various time domain formulations. Figure 2(a) shows both the direct and reflected sound for the plane surface. Figures 2(b)-2(e) zoom in on just the scattered sound for: (b) plane, (c) curved, (d) Schroeder diffuser, Fraunhofer model, and (e) Schroeder diffuser, Kirchhoff model. For the plane surface, the reflection from the center of the surface and the additional delayed edge diffraction waves are as expected. The other surfaces create complex reflection patterns because of waves reflecting from different parts of the surface.

Figure 3 shows the scattered pressure vs frequency for the same surfaces with markers showing the prediction from the frequency-domain models. This demonstrates that the new time-domain models are equivalent and achieve the same accuracy as the frequency-domain formulations. In terms of prediction time, the graphs shown in Fig. 3 where produced, on average, about 12 times quicker with the new time-domain models, compared to using the frequency-domain formulations. 

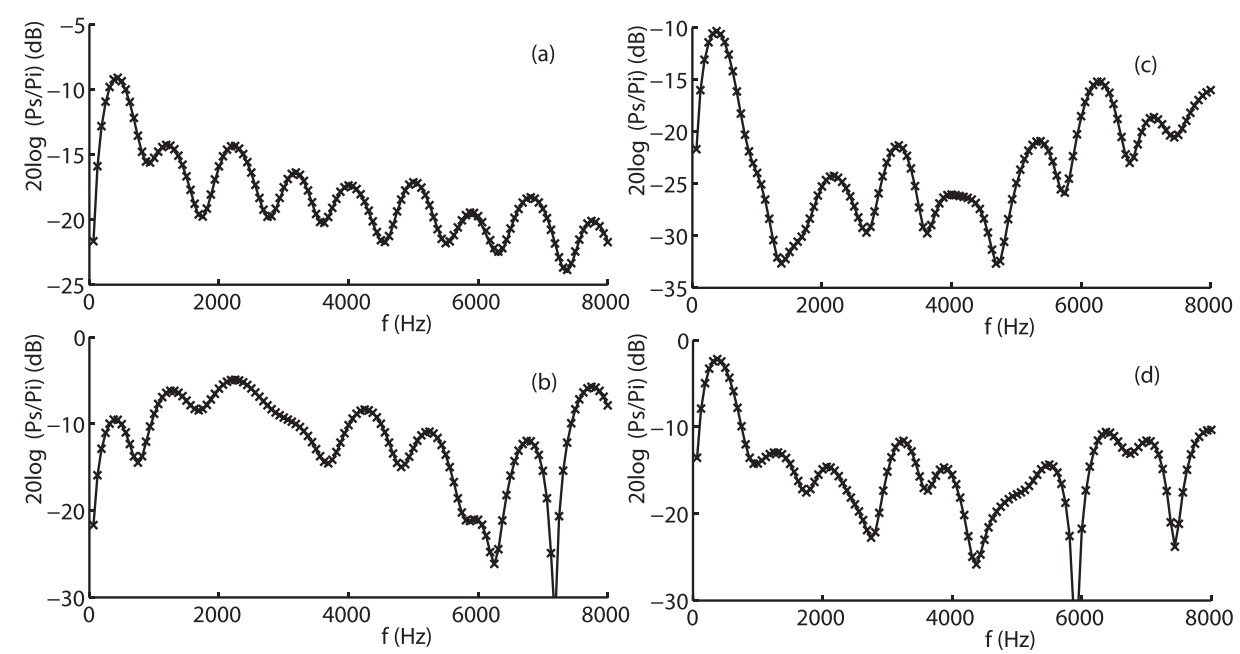

Fig. 3. Ratio of scattered to incident pressure in decibels for the time domain (solid line) and frequency domain models $(\times)$. Surfaces: (a) plane; (b) curved; (c) Schroeder diffuser, Fraunhofer models Eqs. (11) and (13); and (d) Schroeder diffuser, Kirchhoff models Eqs. (12) and (14).

\section{Conclusions}

New formulations have been developed that allow the scattered impulse response from architectural surfaces such as reflectors and diffusers to be rapidly predicted. The methods exploit the Kirchhoff boundary conditions and are analogous to a set of singlefrequency models that are commonly used. The single-frequency models have been shown to be accurate for reflectors and diffusers with low absorption made from materials such as hardwood, metal, and glass reinforced gypsum. The new methods are anticipated to work in the same situations with the advantage that they can more rapidly obtain the impulse response.

FDTD models have a computational cost that scales with the maximum frequency $f$ at $O\left(f^{4}\right)$ because of the combination of using a volumetric mesh and iterative time stepping. In contrast, the new time-domain models use a summation over a surface mesh with computation cost scaling at $O\left(f^{2}\right)$. Consequently, in many scenarios, the new models will be faster than FDTD methods. FDTD will be more accurate, however, because the Kirchhoff boundary conditions are only an approximation of the true surface pressure. The new approaches are intrinsically slower than geometric models because accurate modeling of wave effects requires a higher spatial sampling than is normally used in geometric methods such as ray tracing. The new methods are inherently more accurate at modeling diffraction than geometric methods, however, because the new formulations are wave-based solutions. Example code for the prediction methods used to generate the figures can be downloaded from Ref. 9.

\section{Acknowledgments}

Thanks to Jonathan Hargreaves for his invaluable comments on the method and paper.

\section{References and links}

${ }^{1}$ T. J. Cox and P. D'Antonio, Acoustic Absorbers and Diffusers (Taylor and Francis, London, 2009), pp. 252-288.

${ }^{2}$ M. A. Heckl, "Numerical methods," in Modern Methods in Analytical Acoustics: Lecture Notes, edited by D. G. Crighton, A. P. Dowling, J. E. Ffowcs Williams, M. A. Heckl, and F. A. Leppington (SpringerVerlag, Berlin, 1992), pp. 283-310.

${ }^{3}$ J. A. Hargreaves and T. J. Cox, "A transient boundary element method model of Schroeder diffuser scattering using well mouth impedance,” J. Acoust. Soc. Am. 124, 2942-2951 (2008). 
${ }^{4}$ J. A. Hargreaves, "Time domain boundary element method for room acoustics," Ph.D. thesis, University of Salford, Salford, UK, 2007, pp. 218. Available at http://usir.salford.ac.uk/16604/ (Last viewed February 5, 2015).

${ }^{5}$ J. Sheaffer, M. van Walstijn, and B. M. Fazenda, "Physical and numerical constraints in source modeling for finite difference simulation of room acoustics," J. Acoust. Soc. Am. 135, 251-261 (2014).

${ }^{6}$ U. P. Svensson, R. I. Fred, and J. Vanderkooy, "An analytic secondary source model of edge diffraction impulse responses," J. Acoust. Soc. Am. 106, 2331-2334 (1999).

${ }^{7}$ P. D'Antonio and J. H. Konnert, "The reflection phase grating diffusor: Design theory and application," J. Audio Eng. Soc. 32, 228-238 (1984).

${ }^{8}$ K. Kowalczyk, M. van Walstijn, and D. T. Murphy, "A phase grating approach to modeling surface diffusion in FDTD room acoustics simulations," IEEE Trans. Audio Speech Language Processing 19(5), 528-537 (2011).

${ }^{9}$ http://uk.mathworks.com/matlabcentral/fileexchange/49561-acoustic-modelling-of-surface-scattering-fromreflectors-and-diffusers (Last viewed February 6, 2015). 